\title{
A candidate gene study of one-carbon metabolism pathway genes and colorectal cancer risk
}

\author{
Marcella Martinelli ${ }^{1,2}$, Luca Scapoli ${ }^{1,2}$, Gabriella Mattei $^{1,2}$, Giampaolo Ugolini ${ }^{3}$, Isacco Montroni ${ }^{3}$, \\ Davide Zattoni $^{3}$, Giancarlo Rosati ${ }^{3}$ and Rossella Solmi ${ }^{1,2_{*}}$ \\ ${ }^{1}$ Dipartimento di Istologia, Embriologia e Biologia Applicata, Università di Bologna, Via Belmeloro 8 , \\ 40126 Bologna, Italy \\ ${ }^{2}$ Centro di Ricerca in Genetica Molecolare "Fondazione CARISBO", Bologna, Italy \\ ${ }^{3}$ Dipartimento Emergenza/Urgenza, Chirurgia Generale e dei Trapianti, Università di Bologna, Bologna, Italy
}

(Submitted 2 March 2012 - Final revision received 7 May 2012 - Accepted 18 May 2012 - First published online 16 July 2012)

\begin{abstract}
The risk of colorectal cancer (CRC) may be influenced by aberrant DNA methylation and altered nucleotide synthesis and repair, possibly caused by impaired dietary folate intake as well as by polymorphic variants in one-carbon metabolism genes. A case-control study using seventy-one CRC patients and eighty unrelated healthy controls was carried out to assess the genetic association of fifteen SNP and one insertion in nine genes belonging to the folate pathway. Polymorphism selection was based on literature data, and included those which have a known or suspected functional impact on cancer and missense polymorphisms that are most likely to alter protein function. Genotyping was performed by real-time PCR and PCR followed by restriction analysis. The likelihood ratio statistic indicated that most of the polymorphisms were not associated with the risk of CRC. However, an increased risk of CRC was observed for two variant alleles of SNP mapping on the transcobalamin 2 gene (TCN2): C776G (rs1801198) and c.1026-394T > G (rs7286680). Considering the crucial biological function played by one-carbon metabolism genes, further investigations with larger cohorts of CRC patients are needed in order to confirm our preliminary results. These preliminary results indicate that TCN2 polymorphisms can be a susceptibility factor for CRC.
\end{abstract}

\section{Key words: Colorectal cancer: Folate: Polymorphisms: Transcobalamin 2}

Folate is a water-soluble B vitamin which is essential for DNA synthesis, repair and methylation. Epidemiological analyses have shown that regular consumption of fruit and vegetables - natural sources of folate - correlates with a decreased risk of cancer ${ }^{(1,2)}$. A pregnant woman's intake of one-carbon nutrients can modify her child's risk of incurring tumours, even decades after birth ${ }^{(3)}$. Perturbation of different steps in the folate pathway may induce and accelerate carcinogenesis, as a consequence of DNA instability. There is extensive literature which correlates genetic polymorphisms of folate pathway genes with the occurrence of several diseases and malformations (e.g. neural tube defects, cleft lip/palate, etc.). It is believed that these polymorphisms could alter gene and/or protein functions; however, little is known about the functional impact of each polymorphism and the possible perturbation effect of the overall one-carbon biochemical pathway.

Colorectal cancer (CRC) is currently considered to be the second cause of morbidity and death in the Western world (WHO).
Specific epidemiological and experimental studies have been performed to evaluate the implication of low folate intake in the risk of $\mathrm{CRC}^{(4,5)}$

Some genetic studies have assessed the association between certain nucleotide polymorphisms (SNP) of genes belonging to the folate pathway.

The allelic associations between CRC and the functional C677T (rs1801133) polymorphism of the methylenetetrahydrofolate reductase (MTHFR) gene (which codes for a key enzyme for homocysteine remethylation to methionine) have been extensively studied. The enzyme coded by the $677 \mathrm{~T}$ MTHFR variant allele shows a $60 \%$ activity reduction in vitro ${ }^{(6)}$. In four meta-analysis studies, a modest but statistically significant decrease in the risk of CRC (15-18\%) has been reported for $677 \mathrm{TT}$ homozygotes ${ }^{(7-10)}$. The data were recently confirmed in an independent study ${ }^{(11)}$.

Methionine synthase (MTR) catalyses the vitamin $\mathrm{B}_{12^{-}}$ mediated transfer of a methyl group to convert homocysteine to methionine. Common MTR variants have been reported as

Abbreviations: BHMT, betaine-homocysteine methyltransferase; CBS, cystathionine $\beta$-synthase; CRC, colorectal cancer; CUBN, cubilin; DHFR, dihydrofolate reductase; MTHFD1, methylenetetrahydrofolate dehydrogenase 1; MTHFR, methylenetetrahydrofolate reductase; MTR, methionine synthase; RFC1, reduced folate carrier 1; TCN2, transcobalamin 2 .

*Corresponding author: Dr R. Solmi, fax +39 51 2094110, email rossella.solmi@unibo.it 
being associated with a decrease in CRC risk among non-users of multivitamin supplements ${ }^{(12)}$. The homocysteine remethylation to methionine process also needs betaine-homocysteine methyltransferase (BHMT): a Zn-dependent cytosolic enzyme that utilises betaine as a methyl donor. A slight increased risk of CRC has been associated with the G716A (rs3733890) variant allele of $B H M T^{(13)}$.

Homocysteine trans-sulfuration (the alternative path for homocysteine clearance) is initially catalysed by cystathionine $\beta$-synthase (CBS), a vitamin $\mathrm{B}_{6}$-dependent lyase that generates cystathionine from the condensation of homocysteine with serine. The CBS 844ins68 variant would appear to have a protective effect against CRC because it was less frequently found in patients who developed proximal tumours than in normal control subjects ${ }^{(14)}$.

Dihydrofolate reductase (DHFR) reduces dihydrofolate to tetrahydrofolate, thus allowing its use in methyl group transfers. In patients who do not take multivitamin supplements, two common variants of DHFR SNP have been suggested to be associated with a decreased risk of $\mathrm{CRC}^{(12)}$.

Transcobalamin 2 (TCN2) is a plasma transport protein for cobalamin (vitamin $\mathrm{B}_{12}$ ) and determines vitamin $\mathrm{B}_{12}$ cellular availability. Hazra et al. $^{(15)}$ observed a modest increased risk among carriers of the TCN2 C776G (rs1801198) variant in homo- or heterozygosis for colorectal adenoma.

Although other genes involved in folate uptake, metabolism or distribution have been investigated, no association with CRC risk has been found. Examples are as follows: reduced folate carrier 1 (RFC1/SLC19A1), which is involved in folate uptake ${ }^{(16)}$; methylenetetrahydrofolate dehydrogenase/methylenetetrahydrofolatecyclohydrolase/formyltetrahydrofolate synthetase 1 (MTHFD1), a trifunctional enzyme which catalyses sequential interconversion of tetrahydrofolate derivatives required for purine, methionine and thymidylate synthesis ${ }^{(12)}$; the cubilin gene

Table 1. Patients and control subjects

(Mean values, standard deviations and ranges, or number of subjects)

\begin{tabular}{lccc}
\hline Characteristics & Patients & Controls & $P$ \\
\hline Age (years) & & & \\
Mean & 69 & 58 & 0.64 \\
SD & $13 \cdot 3$ & $12 \cdot 6$ & \\
$\quad$ Range & $29-92$ & $22-83$ & 0.51 \\
Sex $(n)$ & & & \\
Female & 29 & 37 & \\
$\quad$ Male & 42 & 43 & \\
Primary surgery for CRC $(n)$ & & & \\
Yes & 71 & 0 & \\
No & 0 & 80 & \\
Anatomic site $(n)$ & & & \\
Colon & 36 & - & \\
Rectosigmoideum & 16 & - & \\
Rectum & 19 & - & \\
Stage* $(n)$ & & & \\
I & 6 & - & \\
II & 28 & - & \\
III & 22 & - & \\
IV & 15 & - & \\
\hline
\end{tabular}

$\mathrm{CRC}$, colorectal cancer.

* Staging according to the American Joint Committee on Cancer and International Union against Cancer.
$(C U B N)$ that encodes for the intrinsic factor-cobalamin receptor, a transmembrane protein important for the intestinal absorption of cobalamin ${ }^{(12)}$.

In the present study, we investigated fifteen genetic polymorphisms and one insertion mapping in nine genes in order to verify their genetic association with CRC. These nine genes belonging to folic acid and methionine pathways are involved in nucleotide synthesis, methionine metabolism, uptake and distribution of vitamin $\mathrm{B}_{12}$.

\section{Methods}

\section{Sample study}

For the present Italian population-based study, seventy-one consecutively ascertained unrelated CRC patients with confirmed diagnoses were enlisted. The subjects were recruited from the Department of General Surgery at the S. OrsolaMalpighi Polyclinic, Bologna University. The control sample consisted of eighty unrelated volunteers with a healthy general clinical and biochemical assessment. All patient and control information is summarised in Table 1 . The study was approved by the Sant'Orsola-Malpighi General Hospital ethical committee and complied with the Ethical Principles for Medical Research Involving Human Subjects of the Helsinki Declaration. Written informed consent was obtained from all patients and healthy control subjects before being enrolled in the study. DNA extraction from the peripheral whole blood, collected before primary surgery, was performed as described previously ${ }^{(17)}$.

\section{Polymorphisms}

In order to investigate nine folate pathway genes, fifteen SNP and one insertion polymorphism were selected. Polymorphism selection was based on literature data and included those with a known or suspected functional impact, such as SNP associated with cancer or other diseases and missense polymorphisms. Our preference was for those with a minor allele frequency $>0 \cdot 1$ or a moderate degree of linkage disequilibrium between each other.

Genotyping was performed by real-time PCR, using TaqMan assays for BHMT, $C B S$ and $C U B N$ SNP, following the manufacturer's protocol. PCR followed by analysis and separation of fragments by $10 \%$ PAGE stained with ethidium bromide was carried out to investigate MTHFD1, MTR, TCN2, RFC1, MTHFR and DHFR SNP. The presence of the CBS $68 \mathrm{bp}$ insertion (844ins68) was assessed by electrophoresis run on $2.5 \%$ agarose of amplicons. To assess the accuracy of the genotyping outputs, $30 \%$ of the random genotypes were tested in a blind check by a second operator.

The information regarding markers is summarised in Table 2.

\section{Statistical analysis}

The distribution of genotypes in the patient and control groups was tested for deviations from the Hardy-Weinberg equilibrium using Pearson's $\chi^{2}$ test. Case-control association tests were performed using Unphased software version 
Table 2. SNP characteristics, allele count and allelic association test

(Odds ratios and $95 \%$ confidence intervals)

\begin{tabular}{|c|c|c|c|c|c|c|c|c|c|c|c|c|c|c|}
\hline \multirow[b]{2}{*}{ Gene and polymorphism ID } & \multicolumn{3}{|c|}{ Polymorphism characteristics } & \multicolumn{4}{|c|}{ Cases } & \multicolumn{4}{|c|}{ Controls } & \multirow{2}{*}{$\begin{array}{c}\text { Allelic association } \\
\qquad\end{array}$} & \multicolumn{2}{|r|}{ a } \\
\hline & Nucleotide change $^{*}$ & $\begin{array}{c}\text { Location or amino acid } \\
\text { substitution }\end{array}$ & Assay ID/restriction site & A & $\%$ & a & $\%$ & A & $\%$ & a & $\%$ & & OR & $95 \% \mathrm{Cl}$ \\
\hline \multicolumn{15}{|l|}{ BHMT } \\
\hline rs3733890 & $\mathrm{G} / \mathrm{A}$ & Arg239Gln & C_11646606_20 & 88 & 62 & 54 & 38 & 103 & 64 & 57 & 36 & 0.67 & $1 \cdot 11$ & $0.69,1 \cdot 77$ \\
\hline \multicolumn{15}{|l|}{$C B S$} \\
\hline rs234713 & $\mathrm{G} / \mathrm{A}$ & Intron & C_1605453_1 & 104 & 73 & 38 & 27 & 115 & 72 & 45 & 28 & 0.79 & 0.93 & $0.56,1.55$ \\
\hline rs4920037 & $\mathrm{G} / \mathrm{A}$ & Intron & C_1605440_1 & 104 & 79 & 28 & 21 & 125 & 78 & 35 & 22 & 0.89 & 0.96 & $0.55,1.69$ \\
\hline 844ins68 & - & Exon 8 & & 135 & 95 & 7 & 05 & 152 & 95 & 8 & 05 & 0.98 & 0.99 & $0.35,2.79$ \\
\hline \multicolumn{15}{|l|}{ CUBN } \\
\hline rs1907362 & $\mathrm{G} / \mathrm{A}$ & Intron & C_11640657_10 & 131 & 95 & 7 & 05 & 145 & 91 & 15 & 09 & 0.15 & 0.52 & $0 \cdot 20,1 \cdot 31$ \\
\hline rs1801231 & $\mathrm{C} / \mathrm{T}$ & Pro1559Ser & C_26740615_10 & 124 & 87 & 18 & 13 & 143 & 89 & 17 & 11 & 0.58 & $1 \cdot 22$ & $0.60,2.47$ \\
\hline rs1801222 & $\mathrm{C} / \mathrm{T}$ & Phe253Ser & C_2822674_10 & 119 & 85 & 21 & 15 & 131 & 82 & 29 & 18 & 0.47 & 0.82 & $0.40,1.66$ \\
\hline \multicolumn{15}{|l|}{ MTHFD1 } \\
\hline rs2236225 & $\mathrm{G} / \mathrm{A}$ & Arg653Gln & Mspl & 72 & 51 & 70 & 49 & 81 & 51 & 79 & 49 & 0.99 & 1.00 & $0.63,1.57$ \\
\hline rs1950902 & $\mathrm{G} / \mathrm{A}$ & Arg134Lys & BsmAl & 129 & 91 & 13 & 09 & 141 & 88 & 19 & 12 & 0.44 & 0.75 & $0.36,1.58$ \\
\hline \multicolumn{15}{|l|}{ MTR } \\
\hline rs1805087 & $A / G$ & Asp919Gly & HaellI & 122 & 86 & 20 & 14 & 124 & 78 & 36 & 23 & 0.06 & 0.56 & $0.31,1.03$ \\
\hline \multicolumn{15}{|l|}{ TCN2 } \\
\hline rs1801198 & $C / G$ & Pro259Arg & BstNI & 61 & 43 & 81 & 57 & 90 & 56 & 72 & 44 & 0.03 & 1.66 & $1.05,2.62$ \\
\hline rs7286680 & $T / G$ & Intron & HaellI & 73 & 51 & 69 & 49 & 106 & 65 & 56 & 35 & 0.01 & 1.79 & $1 \cdot 13,2.84$ \\
\hline rs10418 & $\mathrm{C} / \mathrm{T}$ & $3^{\prime}$-UTR & Avall & 109 & 77 & 33 & 23 & 133 & 83 & 27 & 17 & 0.17 & 1.49 & $0.84,2.63$ \\
\hline \multicolumn{15}{|l|}{$R F C 1$} \\
\hline rs1051266 & $A / G$ & His27Arg & Haell & 76 & 54 & 66 & 46 & 84 & 53 & 76 & 48 & 0.86 & 0.96 & $0.61,1.51$ \\
\hline \multicolumn{15}{|l|}{ MTHFR } \\
\hline rs1801133 & $\mathrm{C} / \mathrm{T}$ & Ala222Val & Hinfl & 83 & 58 & 59 & 42 & 84 & 52 & 78 & 48 & 0.25 & 0.77 & $0.49,1.21$ \\
\hline \multicolumn{15}{|l|}{ DHFR } \\
\hline rs1643659 & $A / G$ & Intron & $B s t N I$ & 106 & 75 & 36 & 25 & 109 & 68 & 51 & 32 & 0.21 & 0.73 & $0.44,1.20$ \\
\hline
\end{tabular}

$B H M T$, betaine-homocysteine methyltransferase; CBS, cystathionine $\beta$-synthase; CUBN, cubilin; MTHFD1, methylenetetrahydrofolate dehydrogenase 1; MTR, methionine synthase; TCN2, transcobalamin 2; RFC1, reduced folate carrier 1; MTHFR, methylenetetrahydrofolate reductase; DHFR, dihydrofolate reductase.

†An artificial restriction site for Avall was created by using a mutagenic sense primer with a mismatch in the nucleotide sequence. 
3.1.5 within a Windows Vista operative system ${ }^{(18)}$. Genetic association was investigated using both allelic and genotypic tests with a likelihood ratio approach, and statistical significance was assessed by $P$ value. The heterozygote and homozygote OR for the risk allele were calculated in the case of a significant overall genotypic association.

Haplotype association was performed when two or three polymorphisms within a single gene were analysed. First, we carried out a global test to check for any haplotype association, and then we carried out a test on each single haplotype identified.

\section{Results}

A high success rate was obtained during the genotyping stage; in fact, almost $100 \%$ of the genotypes were obtained with the different assays. Genotype distribution was in agreement with the Hardy-Weinberg law.

The allelic association test revealed that allele frequencies in the case study and control groups were similar, with the exception of two polymorphisms in the TCN2 gene (Table 2). Indeed, the variant allele at both C776G (rs1801198) and c.1026-394T > G (rs7286680) loci was more frequent among those in the case study group. The calculated OR were 1.66 (95\% CI 1.05, 2.62; $P$ 0.03) and 1.79 (95\% CI 1.13, 2.84; $P$ 0.01), respectively. However, with regard to the multiple testing issue, none of the association tests exceeded the Bonferroni corrected threshold for statistical significance $(P=0 \cdot 05 / 16=0 \cdot 003)$.

The likelihood ratio statistic used to test genotypic association provided similar results (Table 3). The polymorphic variant rs1801198 showed a significant overall genotypic association $(P=0.01)$ and an increased risk for the homozygote carrier of the rare allele (OR 2.9, 95\% CI 1·1, 7.6). A significant genotypic association was also detected at rs7286680 $(P=0 \cdot 04)$, again with an increased risk for homozygotes (OR 3·1, $95 \%$ CI 1·2, 8·2).
The haplotype association study confirmed the lack of an association with $C B S, C U B N$ and MTHFD1, while it confirmed the association between CRC and polymorphisms at the TCN2 locus. In fact, the overall statistics provided evidence of the association between three marker haplotypes $(P=0.03)$. The estimated OR for each relevant haplotype is reported in Table 4. The haplotypes that included the variant allele at each of the three SNP loci demonstrated a significant increase in the risk of CRC (OR 2.1, 95\% CI 1·1, 3.9).

\section{Discussion}

CRC is among the major causes of morbidity and death in Italy. Both genetic and environmental factors are strongly involved in its aetiology. Epidemiological studies have highlighted the importance of a correct diet and lifestyle, estimating that inappropriate nutrition can be the cause of more than one-third of cancer deaths ${ }^{(1)}$.

Folates are vitamins responsible for a number of processes including DNA stability, synthesis, repair and, not least, methylation. Folate pathway genetic polymorphisms may cause inactivation of the tumour suppressor genes. The hypothesis that a perturbation of the folate pathway could induce carcinogenesis $^{(2)}$ has encouraged researchers to investigate the different enzymes and cofactors involved in this crucial metabolic process as possible cancer susceptibility factors.

For the present investigation, we considered fifteen genetic polymorphisms and one insertion mapping in nine genes of the folate pathway for their possible involvement in colorectal carcinogenesis in the Italian population. Non-synonymous polymorphisms were preferentially selected for the candidate gene investigation because they are more likely to alter protein function. Biochemical data supporting this hypothesis were reported in some cases but in others, no functional data were available.

With the exception of TCN2 polymorphisms, no evidence of an association was found between SNP alleles and the

Table 3. Genotype distribution and overall genotypic association

(Odds ratios and $95 \%$ confidence intervals)

\begin{tabular}{|c|c|c|c|c|c|c|c|c|c|c|c|c|}
\hline \multirow[b]{2}{*}{ Gene } & \multirow[b]{2}{*}{ Polymorphism ID } & \multicolumn{3}{|c|}{ Case } & \multicolumn{3}{|c|}{ Control } & \multirow{2}{*}{$\begin{array}{c}\text { Association } \\
P\end{array}$} & \multicolumn{2}{|c|}{ Heterozygote } & \multicolumn{2}{|c|}{ Homozygote } \\
\hline & & AA & $\mathrm{Aa}$ & aa & AA & $\mathrm{Aa}$ & aa & & OR & $95 \% \mathrm{Cl}$ & OR & $95 \% \mathrm{Cl}$ \\
\hline BHMT & rs3733890 & 29 & 30 & 12 & 32 & 39 & 9 & 0.54 & 1.47 & $0.54,4.00$ & 0.85 & $0.42,1.70$ \\
\hline$C B S$ & rs234713 & 41 & 22 & 8 & 42 & 31 & 7 & 0.58 & 0.73 & $0.36,1.46$ & $1 \cdot 17$ & $0.39,3.52$ \\
\hline$C B S$ & rs4920037 & 42 & 20 & 4 & 52 & 21 & 7 & 0.75 & $1 \cdot 18$ & $0.57,2.46$ & 0.71 & $0.19,2.58$ \\
\hline$C B S$ & 844 ins68 & 64 & 7 & 0 & 73 & 6 & 1 & 0.62 & 1.33 & $0.43,4.17$ & & - \\
\hline CUBN & rs1907362 & 62 & 7 & 0 & 67 & 11 & 2 & 0.46 & 0.69 & $0.25,1.89$ & & - \\
\hline CUBN & rs1801231 & 53 & 18 & 0 & 63 & 17 & 0 & 0.55 & 1.26 & $0.59,2.68$ & & - \\
\hline CUBN & rs1801222 & 52 & 15 & 3 & 53 & 25 & 2 & 0.36 & 0.61 & $0.29,1.29$ & 1.53 & $0.25,9.53$ \\
\hline MTHFD1 & rs2236225 & 16 & 40 & 15 & 21 & 39 & 20 & 0.65 & 1.35 & $0.61,2.95$ & 0.98 & $0.39,2.50$ \\
\hline MTHFD1 & rs1950902 & 60 & 9 & 2 & 61 & 19 & 0 & 0.09 & 0.48 & $0.20,1.15$ & & - \\
\hline MTR & rs1805087 & 53 & 16 & 2 & 50 & 24 & 6 & 0.20 & 0.63 & $0.30,1.32$ & 0.31 & $0.06,1.63$ \\
\hline TCN2 & rs1801198 & 15 & 31 & 25 & 21 & 48 & 12 & 0.01 & 0.90 & $0.41,2.02$ & 2.92 & $1.12,7.58$ \\
\hline TCN2 & rs7286680 & 18 & 37 & 16 & 35 & 36 & 10 & 0.04 & $2 \cdot 00$ & $0.96,4.15$ & $3 \cdot 11$ & $1 \cdot 18,8 \cdot 24$ \\
\hline TCN2 & rs 10418 & 41 & 27 & 3 & 56 & 21 & 3 & 0.28 & $1 \cdot 76$ & $0.87,3.53$ & 1.37 & $0 \cdot 26,7 \cdot 11$ \\
\hline$R F C 1$ & rs1051266 & 22 & 32 & 17 & 19 & 46 & 15 & 0.31 & 0.60 & $0.28,1.29$ & 0.98 & $0.39,2.47$ \\
\hline MTHFR & rs1801133 & 25 & 33 & 13 & 21 & 42 & 18 & 0.45 & 0.66 & $0.32,1.38$ & 0.61 & $0.24,1.52$ \\
\hline DHFR & rs1643659 & 39 & 28 & 4 & 35 & 39 & 6 & 0.39 & 0.64 & $0.33,1.25$ & 0.60 & $0.16,2 \cdot 30$ \\
\hline
\end{tabular}

$B H M T$, betaine-homocysteine methyltransferase; $C B S$, cystathionine $\beta$-synthase; CUBN, cubilin; MTHFD1, methylenetetrahydrofolate dehydrogenase 1; MTR, methionine synthase; TCN2, transcobalamin 2; RFC1, reduced folate carrier 1; MTHFR, methylenetetrahydrofolate reductase; DHFR, dihydrofolate reductase. 
Table 4. Haplotype association analysis between transcobalamin 2 polymorphisms and colorectal cancer*

(Odds ratios and $95 \%$ confidence intervals)

\begin{tabular}{lcccccc}
\hline Haplotype & Cases & Controls & Ca-Freq & Co-Freq & OR & $95 \% \mathrm{Cl}$ \\
\hline C-T-C & 57 & 81 & 0.41 & 0.55 & Ref. & \\
G-T-C & 14 & 21 & 0.10 & 0.14 & 0.95 & $0.44,2.02$ \\
G-G-C & 35 & 24 & 0.25 & 0.16 & 2.07 & $1.12,3.85$ \\
G-G-T & 32 & 22 & 0.23 & 0.15 & 2.07 & $1.09,3.92$ \\
\hline
\end{tabular}

Ca-Freq, case frequency; Co-Freq, control frequency; Ref., reference.

* Haplotypes are combinations of rs1801198, rs7286680 and rs10418 alleles.

occurrence of CRC. Of the three investigated polymorphisms at TCN2, two showed a significantly higher frequency of the variant allele. A significantly increased risk of colon cancer was found in homozygote subjects for the variant alleles, and the OR were $2.9(95 \%$ CI $1.1,7 \cdot 6)$ for C776G (rs1801198) and 3.1 (95\% CI 1.2, 8.2) for c.1026-394T > G (rs7286680).

TCN2 is an essential carrier for the cellular uptake of vitamin $\mathrm{B}_{12}$, a critical cofactor in the remethylation of homocysteine to methionine in mammals ${ }^{(19)}$. Functional studies on TCN2 C776G (rs1801198) have provided evidence that the variant allele may be able to reduce the affinity of TCN2 to vitamin $\mathrm{B}_{12}$, thereby lowering the delivery of vitamin $\mathrm{B}_{12}$ to the tissues $^{(20,21)}$, even if further studies are required to confirm such findings.

Previous investigations have reported conflicting results. Indeed, the TCN2 776G (259Arg) variant allele was found to be relevant to adenoma development ${ }^{(15)}$, while it was not seen to be significantly associated with advanced colorectal adenoma or cancer ${ }^{(13)}$.

In the present study, the functional TCN2 C776G (rs1801198) was not the only TCN2 polymorphism associated with CRC. The intronic SNP c.1026-394T > G (rs7286680) showed a degree of association and OR close to those observed for C776G. Linkage disequilibrium levels between these two polymorphisms were not very close $(D=0.8$, $r^{2} 0.4$ in controls), and this led us to suppose that both of them were independently associated with CRC. However, we cannot exclude the possibility that an unrecognised CRC susceptibility allele(s) could explain the observed data.

The sample size of the present investigation could be considered too small to offer conclusive results regarding the link between the analysed SNP and CRC susceptibility. On the other hand, this is the first significant data to have supported the hypothesis that TCN2 alleles can alter the risk of developing CRC. Although there is only preliminary biochemical evidence available in relation to the functional effects of variant alleles, genetic data support a relationship between TCN2 and CRC. Since CRC continues to be a serious health issue, we strongly believe that further investigations are essential, both to confirm the present findings and to identify possible interactions with the diet and other genes.

\section{Acknowledgements}

This study was supported by grants from the 'Fondazione Enzo Piccinini' (Modena, Italy). We are grateful to Dr Maria
Carmen Biffoni and Dr Daniela Solmi (Laboratorio Analisi Cliniche S. Antonio, Bologna) for their valuable collaboration in supplying blood samples from healthy donors. We are indebted to all the patients who agreed to participate in the study. None of the authors has any personal or financial conflicts of interest. The authors' responsibilities were as follows: M. M. and R. S. conceived and designed the study; G. U., I. M., D. Z., G. R. and R. S. were responsible for the sample collection; M. M., G. M. and R. S. conducted the research; L. S. was responsible for the data analysis and interpretation; M. M. wrote the manuscript; L. S. and R. S. were responsible for the critical revision of the manuscript. All authors read and approved the final manuscript.

\section{References}

1. Doll R \& Peto R (1981) The causes of cancer: quantitative estimates of avoidable risks of cancer in the United States today. J Natl Cancer Inst 66, 1191-1308.

2. Kim YI (2007) Folate and colorectal cancer: an evidencebased critical review. Mol Nutr Food Res 51, 267-292.

3. Ciappio ED, Mason JB \& Crott JW (2011) Maternal onecarbon nutrient intake and cancer risk in offspring. Nutr Rev 69, 561-571.

4. Ulrich CM (2005) Nutrigenetics in cancer research - folate metabolism and colorectal cancer. J Nutr 135, 2698-2702.

5. Davis CD (2007) Nutrigenomics and the prevention of colon cancer. Pharmacogenomics 8, 121-124.

6. Yamada K, Chen Z, Rozen R, et al. (2001) Effects of common polymorphisms on the properties of recombinant human methylenetetrahydrofolate reductase. Proc Natl Acad Sci U S A 98, 14853-14858.

7. Huang Y, Han S, Li Y, et al. (2007) Different roles of MTHFR C677T and A1298C polymorphisms in colorectal adenoma and colorectal cancer: a meta-analysis. JHum Genet 52, 73-85.

8. Hubner RA \& Houlston RS (2007) MTHFR C677T and colorectal cancer risk: a meta-analysis of 25 populations. Int J Cancer 120, 1027-1035.

9. Kono S \& Chen K (2005) Genetic polymorphisms of methylenetetrahydrofolate reductase and colorectal cancer and adenoma. Cancer Sci 96, 535-542.

10. Little J, Sharp L, Duthie S, et al. (2003) Colon cancer and genetic variation in folate metabolism: the clinical bottom line. J Nutr 133, 3758S-3766S.

11. Levine AJ, Figueiredo JC, Lee W, et al. (2010) Genetic variability in the MTHFR gene and colorectal cancer risk using the colorectal cancer family registry. Cancer Epidemiol Biomarkers Prev 19, 89-100.

12. Levine AJ, Figueiredo JC, Lee W, et al. (2010) A candidate gene study of folate-associated one carbon metabolism 
genes and colorectal cancer risk. Cancer Epidemiol Biomarkers Prev 19, 1812-1821.

13. Koushik A, Kraft P, Fuchs CS, et al. (2006) Nonsynonymous polymorphisms in genes in the one-carbon metabolism pathway and associations with colorectal cancer. Cancer Epidemiol Biomarkers Prev 15, 2408-2417.

14. Shannon B, Gnanasampanthan S, Beilby J, et al. (2002) A polymorphism in the methylenetetrahydrofolate reductase gene predisposes to colorectal cancers with microsatellite instability. Gut 50, 520-524.

15. Hazra A, Wu K, Kraft P, et al. (2007) Twenty-four non-synonymous polymorphisms in the one-carbon metabolic pathway and risk of colorectal adenoma in the Nurses' Health Study. Carcinogenesis 28, 1510-1519.

16. Figueiredo JC, Levine AJ, Lee WH, et al. (2010) Genes involved with folate uptake and distribution and their association with colorectal cancer risk. Cancer Causes Control 21, 597-608.
17. Carinci F, Pezzetti F, Scapoli L, et al. (1995) Nonsyndromic cleft lip and palate: evidence of linkage to a microsatellite marker on 6p23. Am J Hum Genet 56, 337-339.

18. Dudbridge F (2008) Likelihood-based association analysis for nuclear families and unrelated subjects with missing genotype data. Hum Hered 66, 87-98.

19. Wuerges J, Garau G, Geremia S, et al. (2006) Structural basis for mammalian vitamin $\mathrm{B}_{12}$ transport by transcobalamin. Proc Natl Acad Sci US A 103, 4386-4391.

20. Afman LA, Lievers KJ, van der Put NM, et al. (2002) Single nucleotide polymorphisms in the transcobalamin gene: relationship with transcobalamin concentrations and risk for neural tube defects. Eur J Hum Genet 10, 433-438.

21. Miller JW, Ramos MI, Garrod MG, et al. (2002) Transcobalamin II $775 \mathrm{G}>\mathrm{C}$ polymorphism and indices of vitamin $\mathrm{B}_{12}$ status in healthy older adults. Blood $\mathbf{1 0 0}$ $718-720$ 